\title{
LESS NOISE, MORE HACKING: HOW TO DEPLOY PRINCIPLES FROM MIT'S HACKING MEDICINE TO ACCELERATE HEALTH CARE
}

\section{Jacqueline W. DePasse}

Resident Physician, Department of Internal Medicine, Massachusetts General Hospital; Consortium for Affordable Medical Technologies

Ryan Carroll

Massachusetts General Hospital Division of Pediatric Critical Care Medicine, Harvard Medical School

Andrea Ippolito

Massachusetts Institute of Technology

\author{
Allison Yost \\ Massachusetts Institute of Technology \\ Data Santorino \\ Lecturer of Pediatrics, Mbarara University of Science and Technology \\ Zen Chu \\ Massachusetts Institute of Technology \\ Kristian R. Olson \\ Medical Director of Consortium for Affordable Medical Technologies, \\ Massachusetts General Hospital, Center for Global Health
}

Medical technology offers enormous potential for scalable medicine- to improve the quality and access in health care while simultaneously reducing cost. However, current medical device innovation within companies often only offers incremental advances on existing products, or originates from engineers with limited knowledge of the clinical complexities. We describe how the Hacking Medicine Initiative, based at Massachusetts Institute of Technology has developed an innovative "healthcare hackathon" approach, bringing diverse teams together to rapidly validate clinical needs and develop solutions. Hackathons are based on three core principles; emphasis on a problem-based approach, cross-pollination of disciplines, and "pivoting" on or rapidly iterating on ideas. Hackathons also offer enormous potential for innovation in global health by focusing on local needs and resources as well as addressing feasibility and cultural contextualization. Although relatively new, the success of this approach is clear, as evidenced by the development of successful startup companies, pioneering product design, and the incorporation of creative people from outside traditional life science backgrounds who are working with clinicians and other scientists to create transformative innovation in health care.

Keywords: Innovation, Health care, Technology, Hackathon, Medicine

\section{HEALTHCARE PRODUCT RE-DESIGN AMID REFORM \& GLOBALIZATION}

Currently, healthcare product development efforts are inefficient, costly, and slow to bring new technologies to market (1). One opportunity to accelerate and encourage significant innovation lies in connecting stakeholders earlier in the development process with a problem-based approach, where they can identify the most urgent clinical needs and create new products, re-architect healthcare systems, and use new databases and tools to improve quality of healthcare delivery.

Medical technology offers enormous potential for scalable medicine - to improve the quality and access in health care while simultaneously reducing cost (2). However, current medical device innovation within companies often only offers incremental advances on existing products, or originates from engineers with limited knowledge of the clinical complexity (3). While this type of technology may generate heavy venture capital investment, it often results in increased treatment costs and designs limited in their population impact (4). To innovate products and services amid the tectonic shifts in healthcare reform and global growth, health institutions and ventures are increasingly embracing the importance of human centered design, or problem-focused technology, pioneered by technology designers.

The Hacking Medicine Initiative, based at Massachusetts Institute of Technology (MIT), uses an innovative "healthcare hackathon" approach to bring diverse teams together to rapidly validate clinical needs and develop solutions. Hundreds of stakeholders self-assemble to form interdisciplinary teams composed of clinicians, patients, engineers, designers, population health experts, and entrepreneurs. Because the product design process is informed by the end-users, the subsequent developments are more likely to fulfill what Everett Rogers in his seminal work, Diffusion of Innovation, defines as criteria for high-impact innovations: that they are better than existing alternatives, relevant to the local context, simple, easily tested, and visible to others (5). Researchers have shown the power of embedding these user-driven innovations in communities that can create connections and "short circuit" the process to increase the speed and effectiveness of disseminating ideas (6). In addition to techniques of design thinking, open innovation (7), and Christensen's Disrupting Healthcare frameworks (8), 


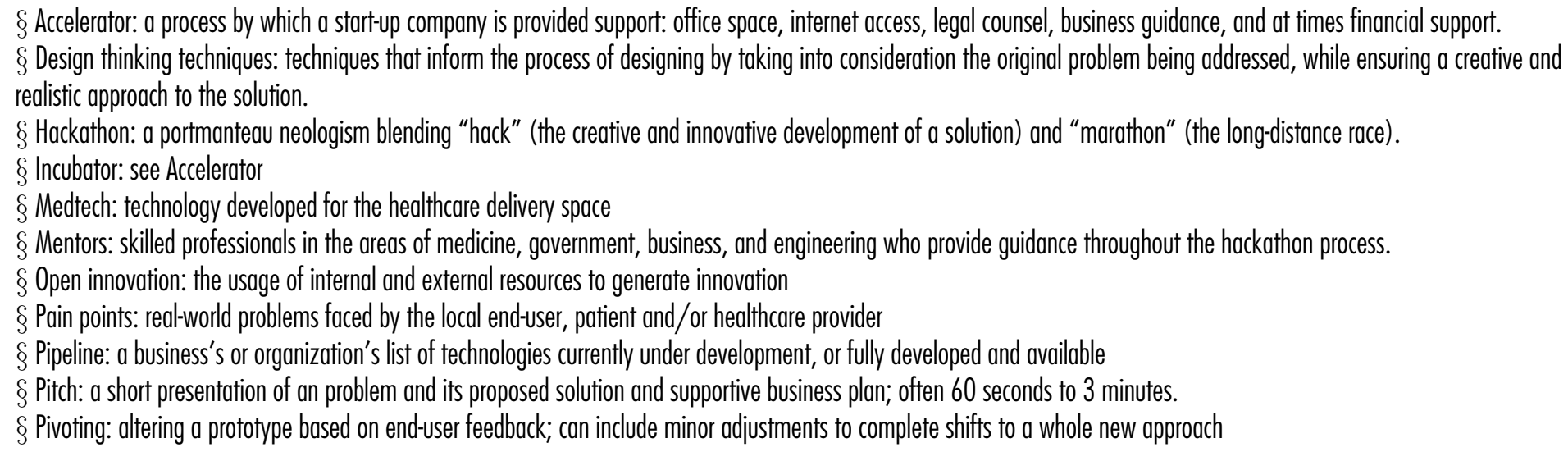

the Hacking Medicine approach emphasizes rapid feedback cycles using a variety of materials, from paper based tools to large clinical databases, to help hone the problem, proposed solutions and business models. In a collaboration with the Massachusetts General Hospital (MGH) Consortium for Affordable Medical Technology (CAMTech) across multiple hackathons in Uganda and India, we have demonstrated that the techniques and processes also translate to create high impact inventions in low and middle income countries (LMIC).

\section{THE HACKING MEDICINE MODEL}

Broadening the principle of "innovation through hacking" to healthcare, Hacking Medicine was formed under the Trust Center for MIT Entrepreneurship in 2011 to translate hacking techniques from computer science to health care (9). Table 1 provides definitions of common terms used in Hacking Medicine and surrounding concepts. The origin of the "hackathon" is thought to have originated from the MIT's Technology Model Railroad Club (TMRC) and the MIT Artificial Intelligence Laboratory in the 1960s (10). Communities of computer programmer enthusiasts and system designers would gather for 24-hour "hacking marathons" where clever solutions were created or improved. These hackathons were popularized in the early 2000 s by the technology community as a forum for quickly developing solutions in short periods of focused innovation. Companies such as Facebook and Google hold hackathons to spur internal innovation. Employees hold 48-hour rapid-fire competitions to "pitch, program, and present a functioning (mobile app)" or another technologic endeavor (11). These companies have described hackathons as being useful for accelerating development efforts and giving staff an opportunity to "think outside the box". In 2012, more than 200 hackathons were held in the United States, hosted by companies varying from 7-Eleven to Foursquare.
MIT Hacking Medicine has extended the hackathon model to address the more complex healthcare environment. Events include the many diverse stakeholders across healthcare regulation, delivery, payment, and product development. While teams tackle both medical device and drug delivery innovation, much of the hacking collaboration involves crowdsourcing the healthcare business models for technology-enabled service delivery across various diseases and health systems. Inventors and existing companies agree to openly collaborate at this early timepoint in the innovation cycle and specifically avoid disclosing intellectual property (10).

The Hacking Medicine group has created a reproducible process for its 48-hour hacks, as shown in Figure 1. First, attendees rapidly pitch "pain points," identifying and quantifying the problems and gaps in health care without yet proposing solutions. These 1-minute pitches are presented in rapid succession, quickly providing a wide array of problems, skill sets, and passions, around which participants organically form teams. Experts in health care, business, and engineering are available as mentors, and provide additional perspectives to the developing teams.

The bulk of the hackathon is comprised of brainstorming sessions and pitches to various health system stakeholders to validate or invalidate the problem, as well as proposed solutions and related business plans. Teams are encouraged to use designthinking techniques while rapidly iterating upon developing solutions over the course of 24 to 48 hours. Rapid feedback from the perspectives of key users - patient, payor, and providerallow open honest feedback while the teams are not yet heavily invested in a particular solution. Therefore, teams can more easily "pivot" and adapt solutions and business models as they learn about the natural history of a disease, the patient journey, the workflows and payment models. Along with product design, teams are encouraged to concurrently develop initial business plans and economics for sustainability and impact. 


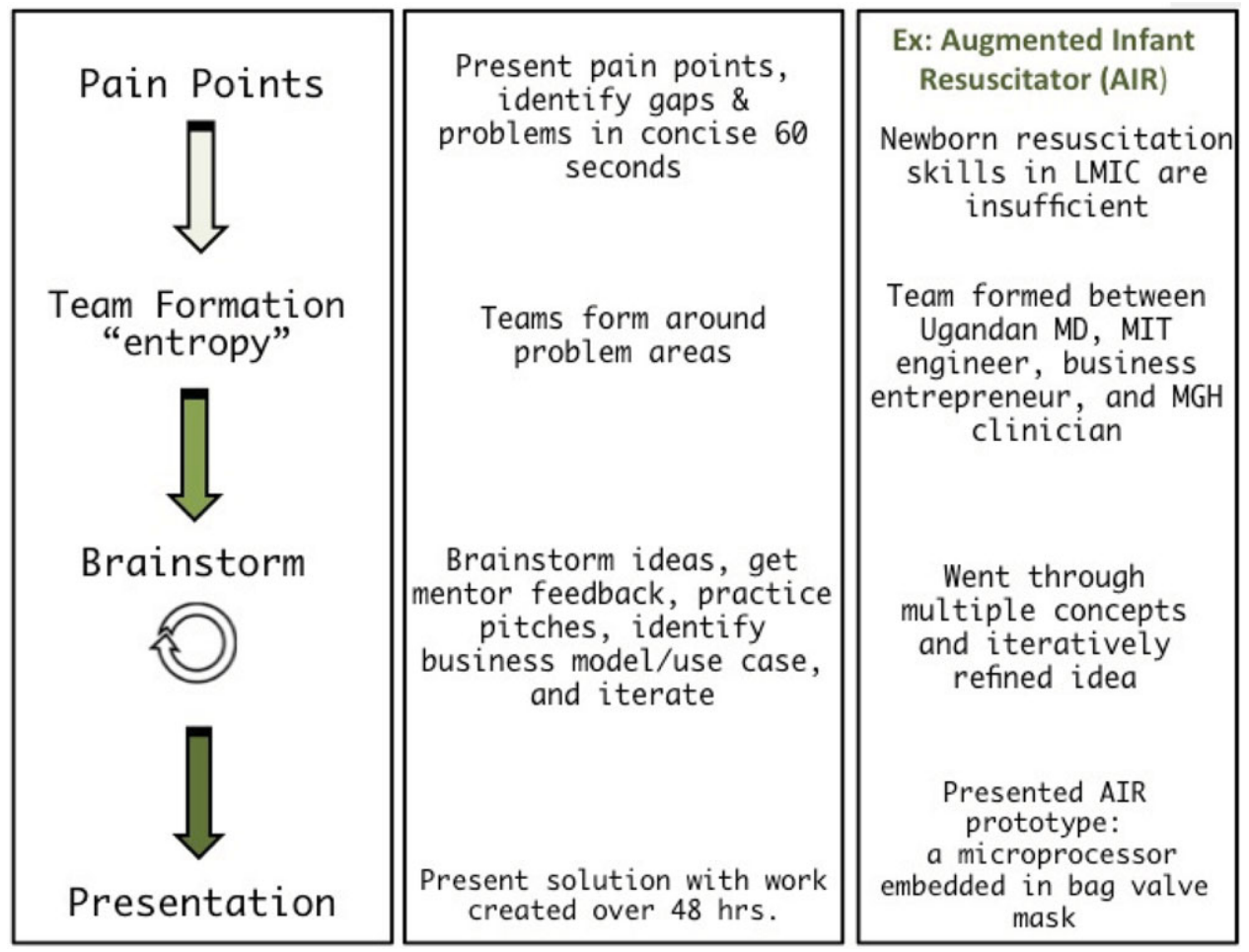

Figure 1. The hacking medicine model and AIR.

Hackathons are competitive events, ending in a final pitch contest with judges who may include healthcare leaders, technologists, entrepreneurs and venture investors. Winning teams convince the judges and fellow attendees of the problem, potential solution and a sustainable business model. Prizes are typically either modest monetary prizes or follow-up consultations with experts and investors, although the prizes vary at each hack.

The hackathon is not meant to be a fully independent process. Medical hackathons are meant to be catalytic processes, and teams are ideally provided access to mentorship networks and funding opportunities such as grants and/or accelerator/incubator programs to propel their concept forward. Teams arising from these events need to enter fertile follow-on processes to develop into successful startup companies.

The Hacking Medicine group has organized over 22 events across the globe, including events in Cambridge, MA and Madrid, Spain. In October 2012, Hacking Medicine partnered with CAMTech to jointly organize a hackathon at MGH in Boston. Since that time, CAMTech and Hacking Medicine have collaborated on four more hackathons, including two in India and one in Uganda. These five international joint efforts have resulted in the development of over 100 innovations, five talent recruitments, five technology development startups, and two CAMTech Innovation Awards of \$100,000 each. The domestic and international hackathons have also stimulated collaborations, job opportunities, and networking connections in each of the locations.

\section{AIR}

The hackathon process and philosophy is exemplified in the development of the Augmented Infant Resuscitator (AIR), a value-based product conceived at the first Hacking Medicine/CAMTech collaborative hackathon at MGH in 2012. The initial "pain point" was identified by Dr. Data Santorino, a pediatrician at the Mbarara University of Science and Technology in Uganda and a national trainer for the international "Helping Babies Breathe" campaign (12). He recognized that newborn resuscitation skills in LMICs are often sub-par, despite training. Yet the need for effective resuscitation is great with over 6 million infants requiring basic infant resuscitation at birth, most of whom are in LMICs (13). Dr. Santorino pitched this challenge at the first joint CAMTech/Hacking Medicine hackathon in Boston with a desire to develop a resuscitation skills enhancement tool.

Dr. Santorino inspired an MIT engineer, a business entrepreneur, and an MGH clinician who joined him to develop an innovative solution. Over the 48 hours of the hackathon, the team discussed multiple concepts, relied on clinical mentors, and iteratively refined their idea. At the end of the hackathon, the team presented a working prototype of AIR, a microprocessor embedded in a bag-valve mask for infants. They won first prize at the event but, more importantly, they continued to work together after the weekend to refine the prototype and business model. The self-selected team applied to and won several awards through MGH and MIT to fund and continue their work. 
Currently, the AIR team has filed Intellectual Property (IP), developed a business model, developed second generation prototypes and completed initial pilot testing of their device in Uganda. AIR has attracted the interest of several medical device companies and received an award from the Saving Lives at Birth Grand Challenges for Development partnership - all in just over 12 months time. Figure 1 demonstrates the extensive influence of the hackathon, using AIR as a model.

\section{PRINCIPLES AND OUTCOMES}

Hackathons provide a unique environment by emphasizing three core principles. First, the hackathon model stresses a problem-based approach, and even encourages participants to "get out of the building" to understand the needs of the clinicians, patients, and other end-users. At the Mbarara University of Science and Technology hackathon in Mbarara, Uganda in August 2013, non-clinical participants spent time in the local hospital inpatient wards and a community health center to more fully understand needs and target solutions. This resulted in the development of teams "hacking" around several different pain points observed firsthand at the hospitals, such as a selfsustaining newborn incubator, an IV infusion measurement tool, and a transport mechanism for pregnant women.

Second, hackathons cross-pollinate a diverse set of thinkers in a "meeting of the minds." The organizers encourage participation from a wide spectrum of disciplines, and highlight the importance of representation of all stakeholders, from clinician to engineer to entrepreneur to end-user. As a result, nontraditional expertise and perspectives contribute to the conversation. By creating an event where technology stakeholders are physically proximate, hackathons serve to condense the innovation timeline from perhaps months to hours, allowing for rapid feedback and productivity.

Third, hackathons place importance on the concept of challenging existing paradigms and "pivoting" frequently. During the short weekend event, the participants are not limited by processes, job descriptions, or funding requirements often found within traditional research and development structures in large organizations. This allows the aforementioned rapid feedback to result in real-time changes and improvements, ensuring that ideas meet the needs of the problem.

AIR is only one of several examples of successful projects that have spawned from hacking medicine events. The weekend events have inspired multiple startup companies addressing a wide range of problems in health care. One example is Pillpack, a startup company with a solution to improve the experience of people with complex medication regimens. This company fills, sorts, and delivers medications in easy-to-use packaging and has progressed within 1 year to receive venture capital funding and expand to over forty U.S. states. Podimetrics is another company developing an insole that collects and transmits data in an effort to prevent complications from diabetic peripheral neuropathy. Smart Scheduling, a third company developed at a hackathon, uses machine learning to help physicians and clinics optimize clinic schedules and predict no-shows.

\section{HACKING GLOBAL HEALTH}

Internationally, hackathons have tremendous potential to catalyze the development of impactful and affordable technology in LMICs. Applying high-income country (HIC) technologies to medical problems within new cultural settings can be fraught with financial, political, and cultural concerns. Starting the process by focusing on local needs, hackathons help to avoid the trap of providing unrealistic care. In addition, special considerations must be made for technologies developed for LMICs where 30 to 48 percent of health care stems from out-of-pocket expenditure (14). It is essential to consider how health care is both delivered and financed in LMICs. Addressing problems proposed by end-users will inevitably take local reimbursement and payer schemes into account. Additionally, hackathons encourage the use of local resources, promoting sustainability and supporting the local economy.

By focusing on the end-user, hackathons also address feasibility issues. It is our experience in LMICs to find crates filled with expensive medical technology such as CT scanners, sitting dormant in the back lot of a hospital in rural Sub-Saharan Africa, and discover that the medical ward lacks basic equipment such as beds and bandages. At the First Global Forum on Medical Devices in Bangkok in 2010, Dr. Margaret Chan, Director-General of the World Health Organization, reported that an estimated 70 percent of high-tech medical equipment in LMICs was non-functional on arrival (15). Approaching local providers and patients to understand their "pain points," helps to inform the design process and avoid wasting time and money developing unsustainable or inappropriate technologies despite good intentions. The hackathon cultivates an approach of end-user feedback and iterative refinement from the onset of development.

Finally, focusing on the end-user from the onset of technology development allows for cultural contextualization. Technologies developed and designed in one cultural context may be translated into a different tool in another geographic setting. The ultrasound, for example, can be viewed as a vital diagnostic tool or a tool for gendercide (16). Tailoring a technology to a specific country or cultural context through hackathons can reduce the risk of having to redesign a technology that was not guided by this process.

\section{CONCLUSION}

Although not all areas of health care are "hackable," there is a great potential to apply development techniques used by high tech companies to health care. Hacking within medicine emphasizes needs-based solutions, cross-pollination of multidisciplinary stakeholders, and "pivoting" or iterative design. 
The Hacking Medicine approach to innovation also borrows philosophies from Silicon Valley and MIT such as design thinking techniques and open innovation. CAMTech, through its work to accelerate medical technology development in LMICs, has recognized Hacking Medicine's approach as a catalytic component of the solution pathway. Although relatively new, the success of this approach is clear, as evidenced by the development of successful startup companies, pioneering product design, and the incorporation of creative people from outside traditional life science backgrounds who are working with clinicians and other scientists to create transformative innovation in health care.

\section{CONTACT INFORMATION}

Jacqueline DePasse (jwdepasse@partners.org)

\section{CONFLICS OF INTEREST}

There is no conflict of interest for any of the authors. The global health hackathons have been supported financially by the Kauffman Foundation, the Bacca Foundation, the Omidyar Network, and the Center for Global Health at Massachusetts General Hospital.

\section{REFERENCES}

1. DiMasi JA, Hansen RW, Grabowski HG Hansen. The price of innovation: New estimates of drug development costs. J Health Econ. 2003;22:151185.
2. Friedman E. The eternal triangle: Cost, access, and quality. Physician Exec. 1991;17:3-6; 8-9.

3. Buntz B. Developing devices that are just what the doctor ordered. Eur Med Device Technol. 2012;3.

4. Linehan J, Pietzsch JB, editors. The medical device innovation process. Washington, DC: InHealth Device and Diagnostics Conference; 2008.

5. Rogers EM. Diffusion of innovations. 5th ed. New York: Free Press; 2003.

6. Hippel Ev. Democratizing innovation. Cambridge, MA: MIT Press; 2005.

7. Chesbrough HW. Open innovation: The new imperative for creating and profiting from technology. Boston, MA: Harvard Business School Press; 2003.

8. Christensen CM. The innovator's dilemma: When new technologies cause great firms to fail. Boston, MA: Harvard Business School Press; 1997.

9. Raymond E. The early hackers. 2000. http://www.catb.org/ esr/ writings/cathedral-bazaar/hacker-history/ar01s02.html (accessed April 1, 2014).

10. Howe J. The rise of crowdsourcing. Boone, IA: Wired Magazine; 2006.

11. Christensen C, Overdorf M. Meeting the challenge of disruptive change. Boston: Harvard Business Review; 2000.

12. American Academy of Pediatrics. Helping babies breathe. www. helpingbabiesbreathe.org (accessed April 1, 2014).

13. Wall SN, Lee AC, Niermeyer S Lee, et al. Neonatal resuscitation in lowresource settings: What, who, and how to overcome challenges to scale up? Int J Gynaecol Obstet. 2009;107(Suppl 1):S47-S62, S63-S64.

14. Mills A. Health care systems in low- and middle-income countries. $N$ Engl J Med. 2014;370:552-557.

15. World Health Organization. Global forum to improve developing country access to medical devices. 2010. http://www.who.int/mediacentre/ news/notes/2010/medical_devices_20100908/en/ (accessed April 1, 2014).

16. Muller-Rockstroh B. Appropriate and appropriated technology: Lessons learned from ultrasound in Tanzania. Med Anthropol. 2012;31:196-212. 